\title{
Development of Data Acquisition Console and Web Server Using Raspberry Pi for Marine Platforms
}

\author{
Aditya Thapliyal \\ PG Student, Dept of Electronics, Defence Institute of Advanced Technology, DIAT (DU) \\ Girinagar, Pune, India \\ E-mail: adityathapliyal@gmail.com \\ Dr. CRS Kumar \\ Associate Professor, HoD-Computer Engineering, Defence Institute of Advanced Technology, DIAT (DU) \\ Girinagar, Pune, India \\ E-mail: suthikshnkumar@diat.ac.in
}

\begin{abstract}
Marine vessels in today's age are fitted with a number of state of the art systems required for their smooth operation. The compartments which house such systems along with the restricted compartments onboard ships such as the ships galley, dry rations store, cold rooms, battery compartments etc are required to be monitored on real time basis for temperature, pressure, humidity for detecting various hazards like fire, flooding etc. In addition, military platforms also need to monitor compartments such as the armory and magazines to avoid damage to munitions and prevent unauthorized access. The present project aims to develop a proof of concept prototype real time parameter monitoring and motion detection system for critical/restricted compartments on marine platforms with data logging capability.

Various sensors forming a sensor suite have been interfaced to the Raspberry Pi board, forming the Data Acquisition Console which is the nodal control center. As most marine vessels are fitted with a shipboard Local Area Network, the project utilizes this existing network for relaying data. The console is placed in the compartment where parameters are to be monitored and the measured data is acquired and transferred via wireless (using Access Points (APs) operating on Wi-Fi/ 802.11 network) or via wired connectivity with the nearest switch and be accessed by concerned personnel at various nodes/ computer on the . The performance of the DAC was successfully ascertained by comparison of sensor performance with other independent sensor readings. The measurement errors were found to be within the permissible accuracy limits of the sensors. Motion detection was achieved by using PIR motion The probability of detection $\left(\mathrm{P}_{\mathrm{d}}\right)$ for the motion sensor was calculated by conducing iterative motion tests with favorable results. Data is displayed in a web-based dashboard Graphical User Interface. Further, provision has also been made to set visual alarms whenever a particular sensor reading crosses a pre-designated safe limit.
\end{abstract}

Index Terms-Raspberry Pi, Data Acquisition Console, Web Server, MySQL server, Python, Passive Infrared (PIR).

\section{INTRODUCTION}

Although present marine vessels incorporate various sensor systems for monitoring of critical parameters such as temperature, pressure humidity etc, they are generally expensive and do not allow for easy customization by the end user post purchase. Also, most of such systems have the data displayed/available only is specific locations. Keeping this view in mind, the purpose of this paper is to develop a low cost alternative to the existing sensor systems with the capability to allow user customization such as setting warning, threshold limits etc. and also access to the sensor measurements in all compartments which have computer nodes connected on the ships LAN.

As at present, monitoring of parameters in cold rooms, armoury, magazines, battery compartments etc is done by physically taking rounds of such compartments, the project provides remote monitoring of all such parameters including a first of its kind motion detection feature for such compartments. Thus, the project potentially allows for better manpower management onboard as it mitigates the requirement of taking physical rounds of restricted compartments.

Modern marine vessels are installed with a LAN to facilitate fast and efficient flow of information between the different offices/ compartments onboard the ship to carry out day-to-day functioning. Also, ships already have from a few to several hundred kilometers of cabling onboard and installation of a new system/ equipment/sensor suite entails laying of new cables for the system which is a tedious task. Therefore, utilization of the existing Ethernet network for data distribution to various locations across the ship, would save an extensive task of cabling across decks and bulkheads and thereby preserve the watertight and gas tight integrity of the vessel. 
Generally the Ethernet network onboard is distributed all across the ship with LAN switches placed at various locations. This network is lightly loaded with generally less than 100 nodes connected on the ship's network and several ports are available for use in the LAN switches. Hence, there is a great scope to utilize this existing network for connecting the DAC (Raspberry Pi board with sensors). The DAC can be placed in the compartment where parameters are to be monitored and the data parameters can be acquired and ported/ transferred via wireless (using Access Points (APs) operating on Wi-Fi/ 802.11 network) or via wired connectivity with the nearest switch and thus can then be accessed by all the nodes at various locations on the ships network. The DAC nodes monitoring and collating parameters in restricted compartments are mostly placed near the watertight doors for better connectivity with the AP in corridor. Block diagram of the DAC design is indicated in Fig. 1.

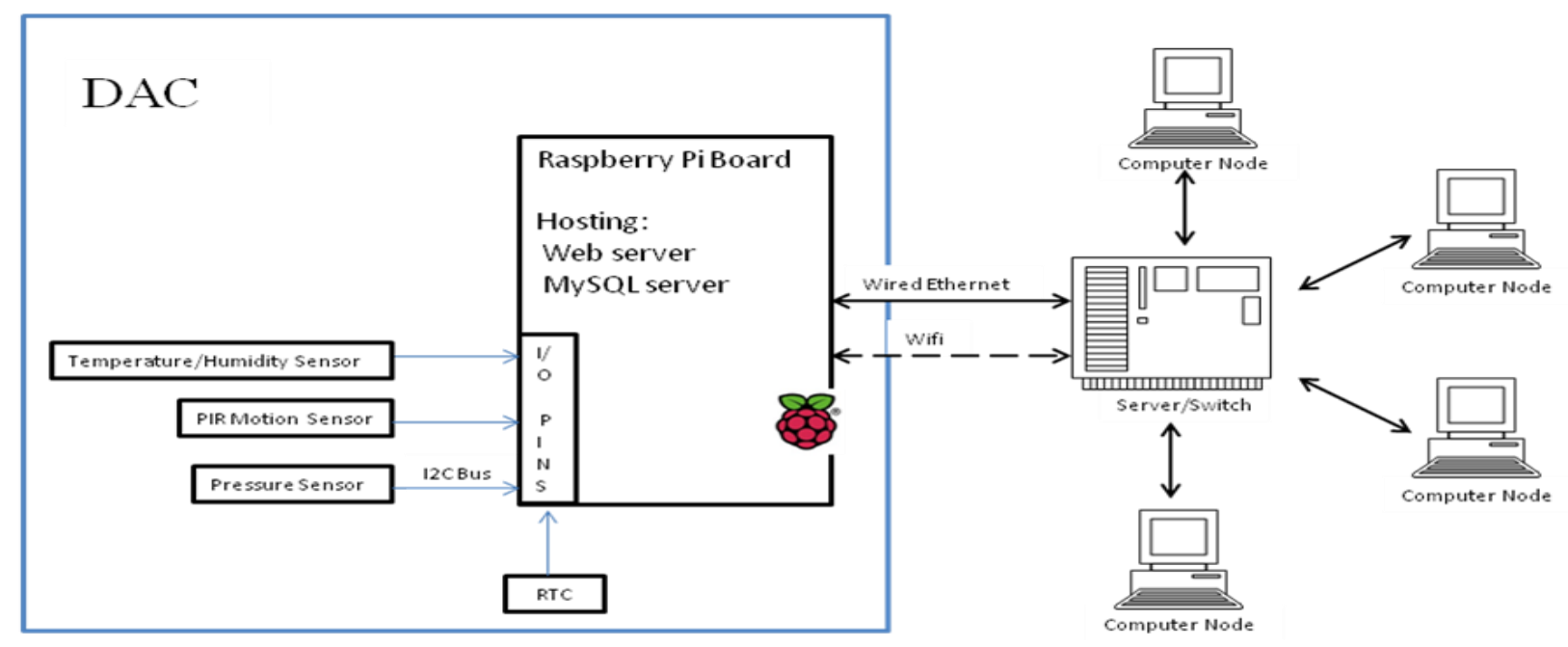

Fig.1. Block Diagram of Data Acquisition Console

The data acquisition development and logging is implemented using open source software. Python language has been used to program polling of sensor readings and logging of parameters on the microprocessor. The microprocessor board is capable of hosting Apache web server and MySQL server applications. Data collected from sensors is logged in a MySQL database and hosted on a web based dashboard GUI using PHP scripting language. The GUI is accessible to the computer nodes on the ships LAN.

The flow of the paper is as follows: Section II covers the Problem Statement/Motivation, Section III covers the components which comprise the DAC and sensor suite, Section IV emphasizes on the development of the DAC, Section V highlights DAC performance and at the end, Section VI concludes the paper.

\section{Problem Statement /MotiVAtion}

To develop an integrated proof of concept DAC prototype to monitor and log critical parameters and motion detection for unauthorized access in restricted compartments onboard and make data available on ships network.

\section{Objectives of the project:-}

(a) Real time monitoring of temperature and humidity in temperature sensitive areas/ restricted areas such as engine room/ boiler rooms, magazines, cold rooms, battery compartments, critical machinery compartments etc.

(b) Monitor access to restricted compartments onboard by aid of IR motion detector such as magazines, armory, onboard Freezers/cold rooms etc.

(c) Remote access/ through web-based site available on the Ship's LAN.

(d) Data logging of measured readings for causative analysis.

(e) Introduce pressure sensor which can be used for validation or also as a means of redundant readings during pressure testing of ship citadels. The sensor can also be used to calculate the altitude based on the pressure output and thus can also be used as an altimeter for aircrafts.

\section{DAC COMPONENTS}

At the heart of the DAC is the Raspberry Pi microprocessor board which has been interfaced with the various sensors required for this project. Visual alarms in the form of LEDs have also been interfaced and are triggered when a sensor reading goes beyond a predefined limit. The sensors are interfaced via the General Purpose Input Output (GPIO) pins and the Inter-Integrated Circuit (I2C) bus lines.

\section{A. Raspberry Pi Microprocessor}

Raspberry $\mathrm{Pi}$ is a microprocessor based single-board computer running Linux, it can be programmed in $\mathrm{C}++$, Java, Python and other popular languages. Block Diagram 
of the Raspberry Pi 2 board is shown in Fig. 2.

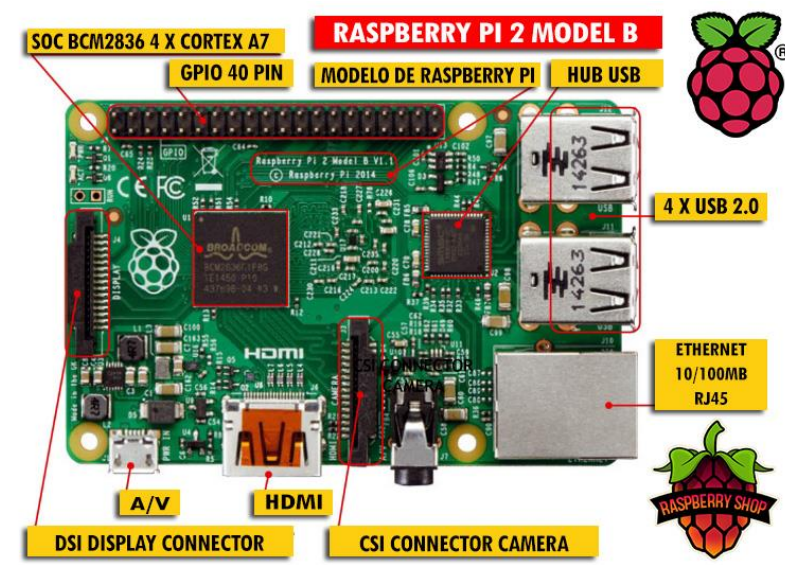

Fig.2. Block Diagram of Raspbery Pi 2 Board

The Raspberry Pi (and its upgraded version, Raspberry Pi 2) is a series of Advanced RISC Machines (ARM) powered, credit card sized single-board computers made in the UK by the non-profit Raspberry Pi Foundation organization. It needs an Operating System (OS) to work and the OS along with storage is provided from an external SD card. The board can be connected to a network via an Ethernet Cable [1]. It can drive HDMI displays, process mouse, keyboard, and camera inputs, control hardware, connect to the Internet and more.

The Raspberry Pi 2 Model B is the second generation Raspberry Pi. It is capable of 6 times the processing capacity of previous models. This second generation Raspberry $\mathrm{Pi}$ has an upgraded Broadcom BCM2836 processor, which is a powerful ARM Cortex-A7 based quad-core processor that runs at $900 \mathrm{MHz}$. The board also features an increase in memory capacity to 1 Gbyte.

The $\mathrm{Pi}$ is preferred for this project as it has an inbuilt LAN port as well as 4 USB ports and can connect easily to a wired/wireless network without the addition of any external electronic components. It supports a large number of input output peripherals making it the perfect platform to interface many devices [5][6]. It can also be used as a remote server for running the application GUI relevant for this project. The $\mathrm{Pi}$ is an incredibly powerful platform in a very small package and is user friendly.

\section{B. Sensors}

\section{DHT 22}

The acquisition of temperature and humidity data has been implemented using low cost DHT 22 sensor which is readily available in the market. DHT22 provides a calibrated digital signal output. It utilizes exclusive digital-signal-collecting-technique and humidity sensing technology, assuring its reliability and stability. Its sensing elements are connected with 8-bit single-chip computer. Every sensor of this model is temperature compensated and calibrated in accurate calibration chamber and the calibration-coefficient is saved in type of program in One Time Programmable (OTP) memory, when the sensor is detecting, it will cite coefficient from memory. Its small size \& low consumption \& long transmission distance (20m) enable DHT22 to be suited in all kinds of harsh application occasions [7]. The DHT sensor is made of two parts, a capacitive humidity sensor and a thermistor. There is also a very basic chip inside that does some analog to digital conversion and outputs a digital signal with the temperature and humidity.DHT22 sensor is shown in Fig 3.

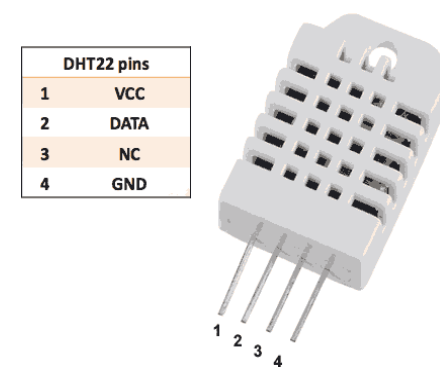

Fig.3. DHT 22 Temperature Sensor

\section{PIR Sensor HC-SR 501}

A Passive Infrared (PIR) sensor can be used to detect these passive radiations. It is a pyro electric sensor sensitive to IR. (long wave IR $: 8-15 \mu \mathrm{m}$ wavelength). This is the thermal imaging region, in which sensors can obtain a completely passive image of objects only slightly higher in temperature than room temperature When an object (human or animal) emitting infrared radiations passes through the field of view of the sensor, it detects the change in temperature and therefore can be used to detect motion. The module has an on-board pyroelectric sensor, conditioning circuitry and a dome shaped Fresnel lens to focus the IR radiation onto the sensor. The output of the sensor will be high when objects enter the sensing range, and automatically turn to low when object leave the coverage range.PIR sensor is shown in Fig 4.

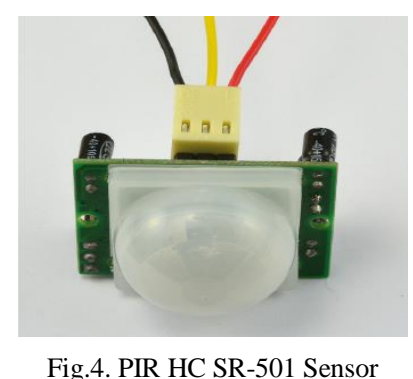

The module has a rectangular window with two sub probes 1 and 2 located at two ends of the rectangle. Each probe is made of a special material that is sensitive to IR. When the sensor is idle, both slots detect the same amount of IR, the ambient amount radiated from the room or walls or outdoors. When a warm body like a human or animal passes by, it first intercepts one half of the PIR sensor, which causes a positive differential change between the two halves. When the warm body leaves the sensing area, the reverse happens, whereby the sensor generates a negative differential change. These change pulses are what is detected [8]. The Fresnel lens condenses and 
focuses light, providing a larger range of IR to the sensor.The lens is made of polyethylene and is transparent to IR radiation. Fresnel lens is shown in Fig 5.
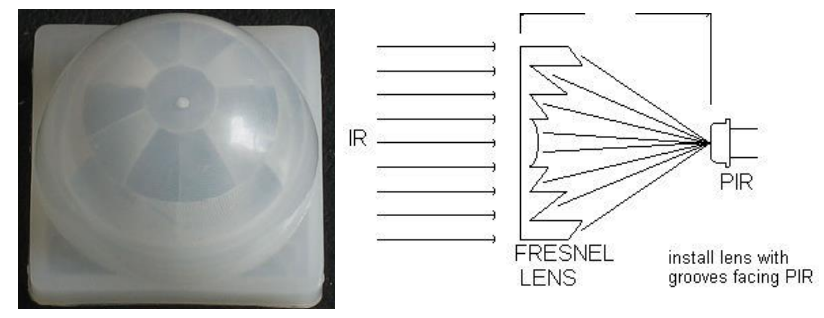

Fig.5. PIR HC SR-501 Sensor lens

\section{BMP 180 Digital Pressure Sensor}

The BMP180 from Bosch is a high precision digital pressure sensor for consumer applications. The ultra-low power, low voltage electronics of the BMP180 is optimized for use in mobile phones, PDAs, GPS navigation devices and outdoor equipment. The BMP180 is designed to be connected directly to a processor of a device via the $\mathrm{I} 2 \mathrm{C}$ bus. This precision sensor is a low-cost sensing solution for measuring barometric pressure and temperature. As pressure changes with altitude it can also be used as an altimeter. This board is $5 \mathrm{~V}$ compliant and includes a $3.3 \mathrm{~V}$ regulator and an $\mathrm{I} 2 \mathrm{C}$ level shifter circuit so this sensor can be used safely with $5 \mathrm{~V}$ logic and power. The pressure and temperature data has to be compensated by the calibration data of the E2PROM of the BMP180.The sensor is based on piezo-resistive technology for EMC robustness, high accuracy and linearity as well as long term stability [9]. The BMP180 consists of a piezo-resistive sensor, an analog to digital converter and a control unit with E2PROM and a serial I2C interface. The sensor is a piezoresistive sensor fabricated using MEMS technology [10]. BMP pressure sensor is shown in Fig 6.

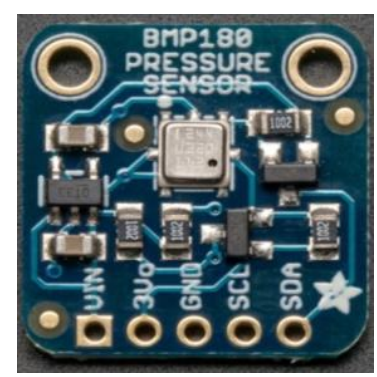

Fig.6. BMP 180 Sensor

\section{DEVELOPMENT OF DAC}

All the connections required to interface the Raspberry $\mathrm{Pi}$ and the sensor suite is set up according to the circuit. Python script is written to initialize the GPIO pins of the Raspberry Pi in order to poll the sensors for measurement. The board is further programmed to log the measurement reading and store it locally on a file. The sensor data is viewed as a web based dashboard GUI and can be accessed by all the connected nodes on the LAN. The data is displayed in a $2 \mathrm{D}$ graph format with set limit lines for each sensor reading. The GUI webpage is a dynamic page written in PHP script and is refreshed periodically.

The GUI dashboard also has links to view the data in a tabular format as well as to view the list of the backed up .csv files in case the files need to be accessed for future reference/ inquiry. The flow of the project development is shown in Fig 7.

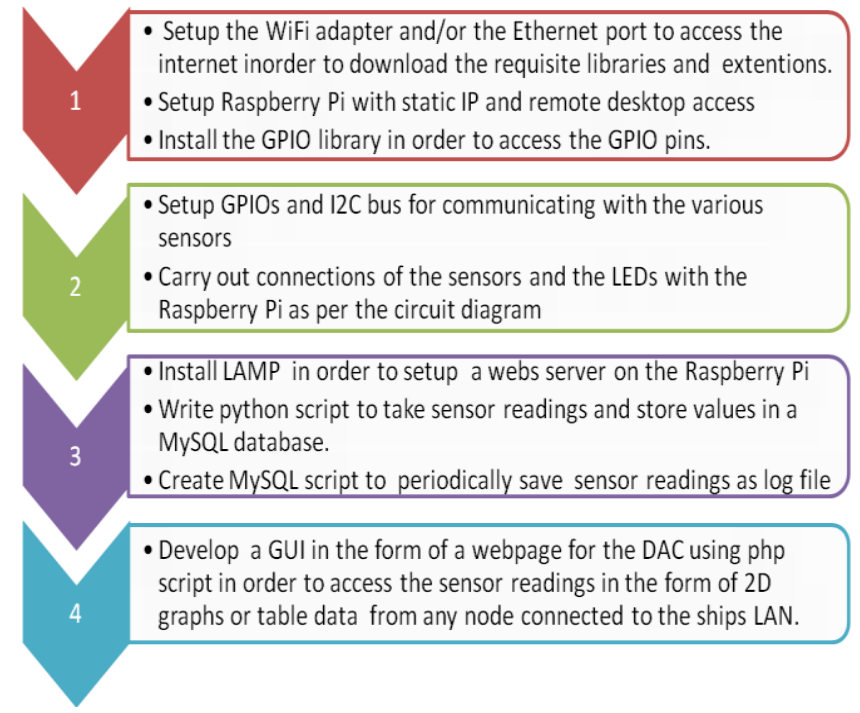

Fig.7. Flow of project with milestones

\section{A. Hardware Implementation}

A lot of research has been undertaken to study the propagation of wireless signals onboard ships. Several experiments have been undertaken in this regard. The 2.4 $\mathrm{GHz}$ ISM frequency band was selected as it is a widely used standard. Each shipboard environment was measured using a common procedure. Overall, conclusions based on the experiments indicated that wireless communications between adjacent rooms are possible in presence of non-conductive materials in the common bulkhead. Watertight doors are the main source of radio leakage between adjacent rooms. [2][3][4]. Hence, wireless communications is indeed possible within the ship, and the DAC must be placed as close to the AP (Access Point) as possible to ensure reliable communication with minimal signal path loss.

In addition to the sensors, the DS-1307 Real Time Clock (RTC) module has also been interfaced with the DAC in order to keep track of current time when the system is not connected to the internet [11]. It is required for data timestamp.The detailed schematic layout of the $\mathrm{DAC}$, i.e. the Raspberry $\mathrm{Pi} 2$ board, the sensors, indication LEDs and the RTC module along with a legend of the layout are shown in Fig 7 and Table 1 respectively. 


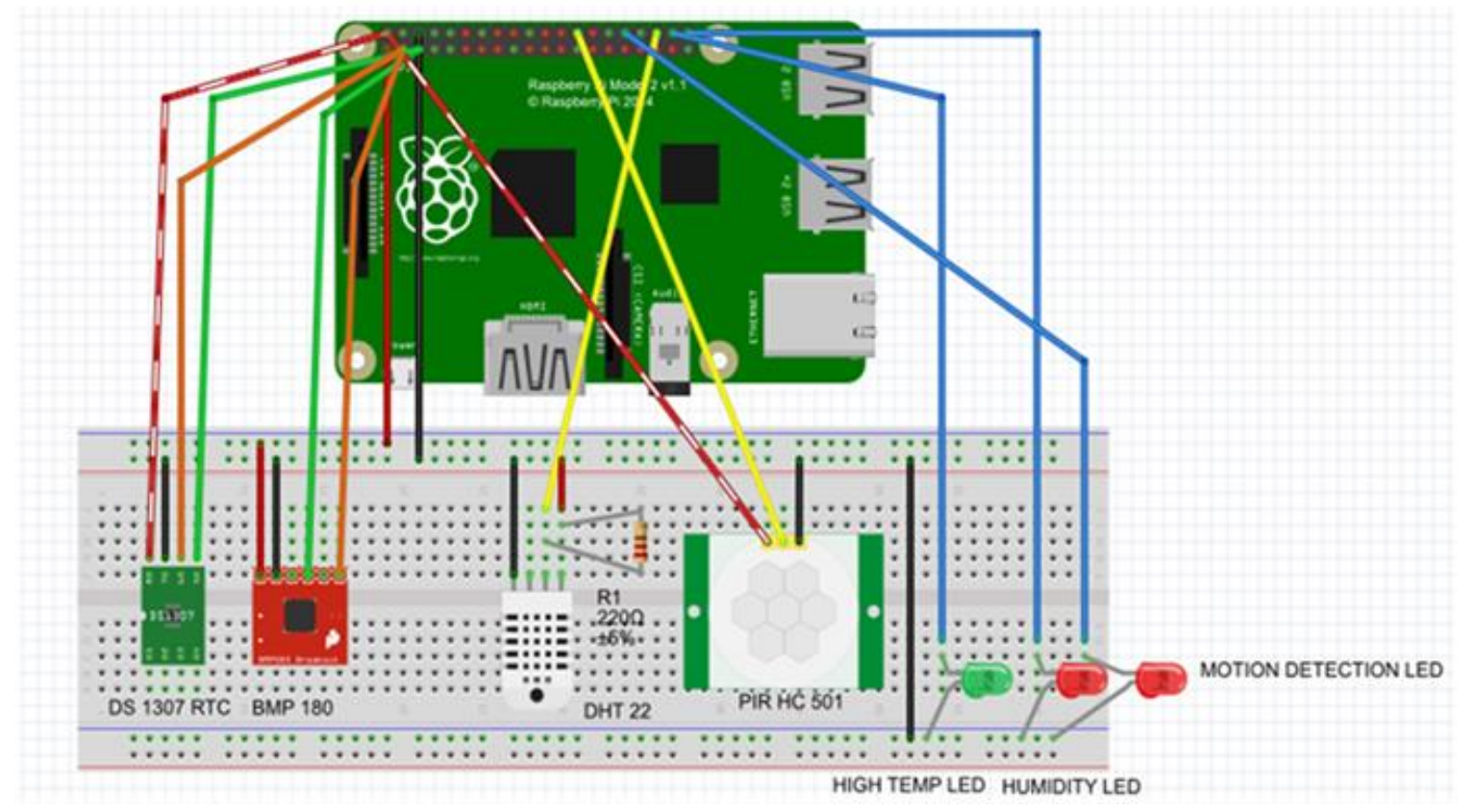

Fig.8. DAC Schematic Layout

Table 1. Legend of Layout

\begin{tabular}{|l|l|}
\hline Colour code & \multicolumn{1}{|c|}{ Description } \\
\hline & 3V DC Supply \\
\hline & Ground \\
\hline & GPIO 2 SDA1 I2C \\
\hline & GPIO 3 SCL 12C \\
\hline & GPIO 12 and 24 for LEDS \\
\hline & $\begin{array}{l}\text { GPIO 7 : Data for PIR sensor } \\
\text { GPIO 16 Data for DHT 22 sensor }\end{array}$ \\
\hline & 5V DC supply for PIR sensor and RTC \\
\hline
\end{tabular}

\section{B. Software Implementation}

\section{Installation of requisite software and libraries}

Once the Pi is setup on the network and has access to the internet, the existing OS firmware is updated to the latest build. To do so we type the following commands sudo apt get update and sudo apt get upgrade in the terminal window. The former updates the list of available packages and their versions and the latter actually installs newer versions of the packages.

To access the GPIO pins on the Raspberry Pi, the GPIO library is loaded onto the $\mathrm{Pi}$ via following commands entered in the terminal window.

\section{sudo apt-get install python-pip}

sudo pip install RPi.GPIO

\section{Setting up static IP and remote access}

First thing is to power up the Raspberry $\mathrm{Pi}$ after connecting the provided Wi-Fi dongle adapter. After logging in, we need to setup the Raspberry pi for a static IP address in order to run it as a server. Static IP can be provided by adding the Raspberry $\mathrm{Pi}$ as an exception to the DHCP allocation table in the router settings or can also be done by on the Raspberry Pi itself by configuring the interfaces file using the command sudo nano letc/network/interfaces.

After setting up static IP, one can remotely gain access to the command line of a Raspberry $\mathrm{Pi}$ from another computer on the same network using Secure Shell (SSH) [12]. The popular SSH client used for windows is called PuTTY and is available online for free. Alternatively, the Raspberry pi can also be controlled using built-in remote desktop connection application on Windows platforms using the $x r d p$ software loaded on the $\mathrm{Pi}$. The $x r d p$ software allows the Raspberry Pi to communicate with another computer via Remote Desktop Protocol (RDP) [13]. The $x r d p$ software can be installed on the Pi using command sudo apt-get install $x r d p$ in the terminal window.

\section{Setting up web server on Raspberry Pi}

LAMP is an acronym that stands for LinuxApacheMySQLPHP, these are the components required to run a Dynamic HTML webpage and these entail the first few steps to build a LAMP web server on a Raspberry Pi. It is an open-source Web development platform, also called a Web stack that uses Linux as the operating system, Apache as the Web server, MySQL as the RDBMS and PHP as the object-oriented scripting language. The stack can be downloaded on the Raspberry Pi (connected to the internet) using the command

sudo apt-get install apache2 php5 libapache2-mod-php5 mysql-server mysql-client php5-mysql.

For easier administration of MySQL, phpMyAdmin has been used. It is a free software tool written in PHP scripting language, intended to handle the administration of MySQL over the Web and supports a wide range of 
operations on MySQL [14]. It is basically a GUI version and is an alternative to the Command Line Interface (CLI). After installation of LAMP server, phpMyadmin software is downloaded onto the Raspberry pi using command sudo apt-get install phpmyadmin.. Python language is used program to read the sensor data. MySQL is used to $\log$ the measurements in a database and the log values are displayed on a webpage using the PHP script for display of dynamic data [15][16]. Snapshots of the DAC dashboard are shown in Fig 9 and 10 respectively.

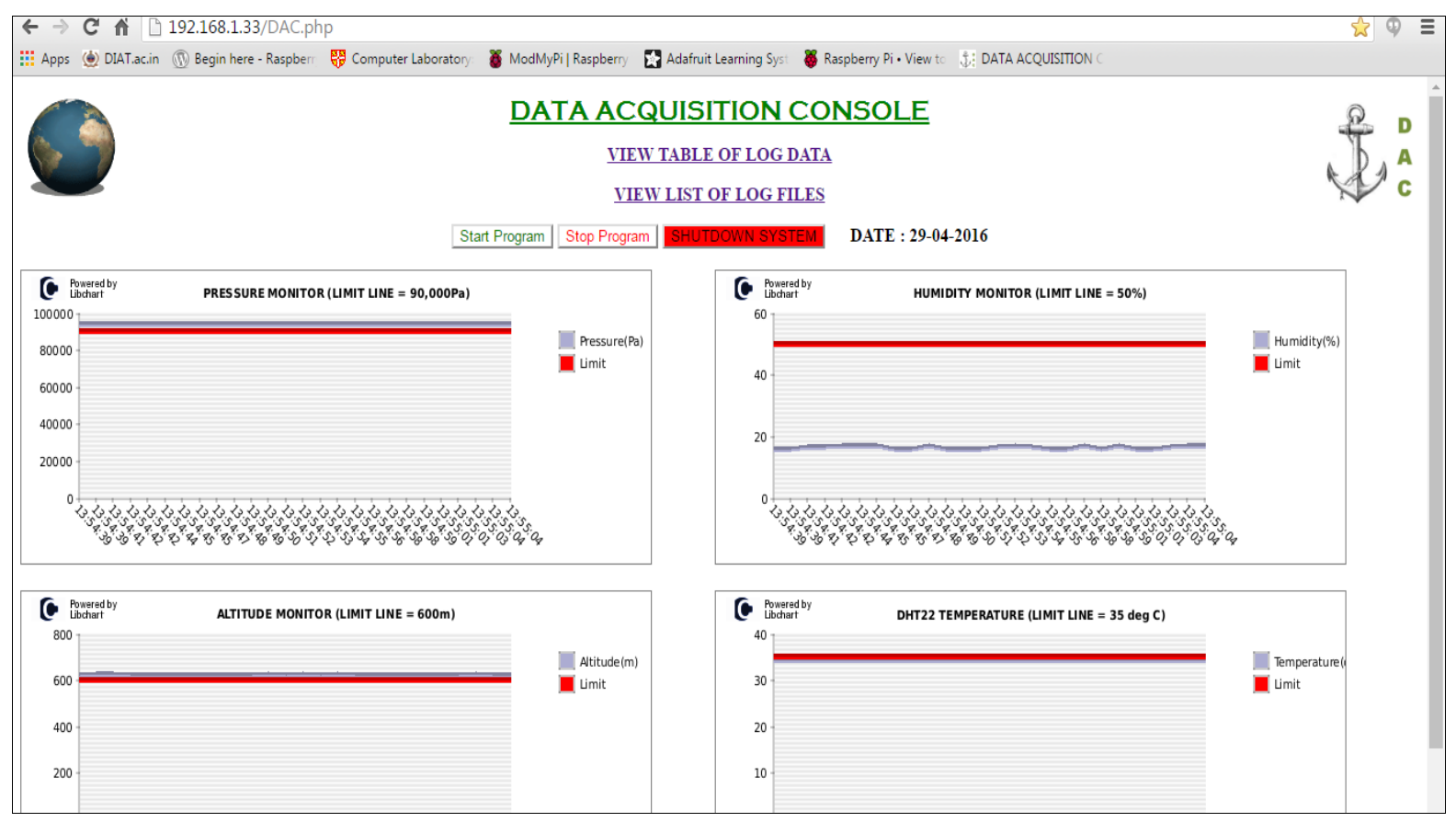

Fig.9. DAC Dashboard - 2D graph view

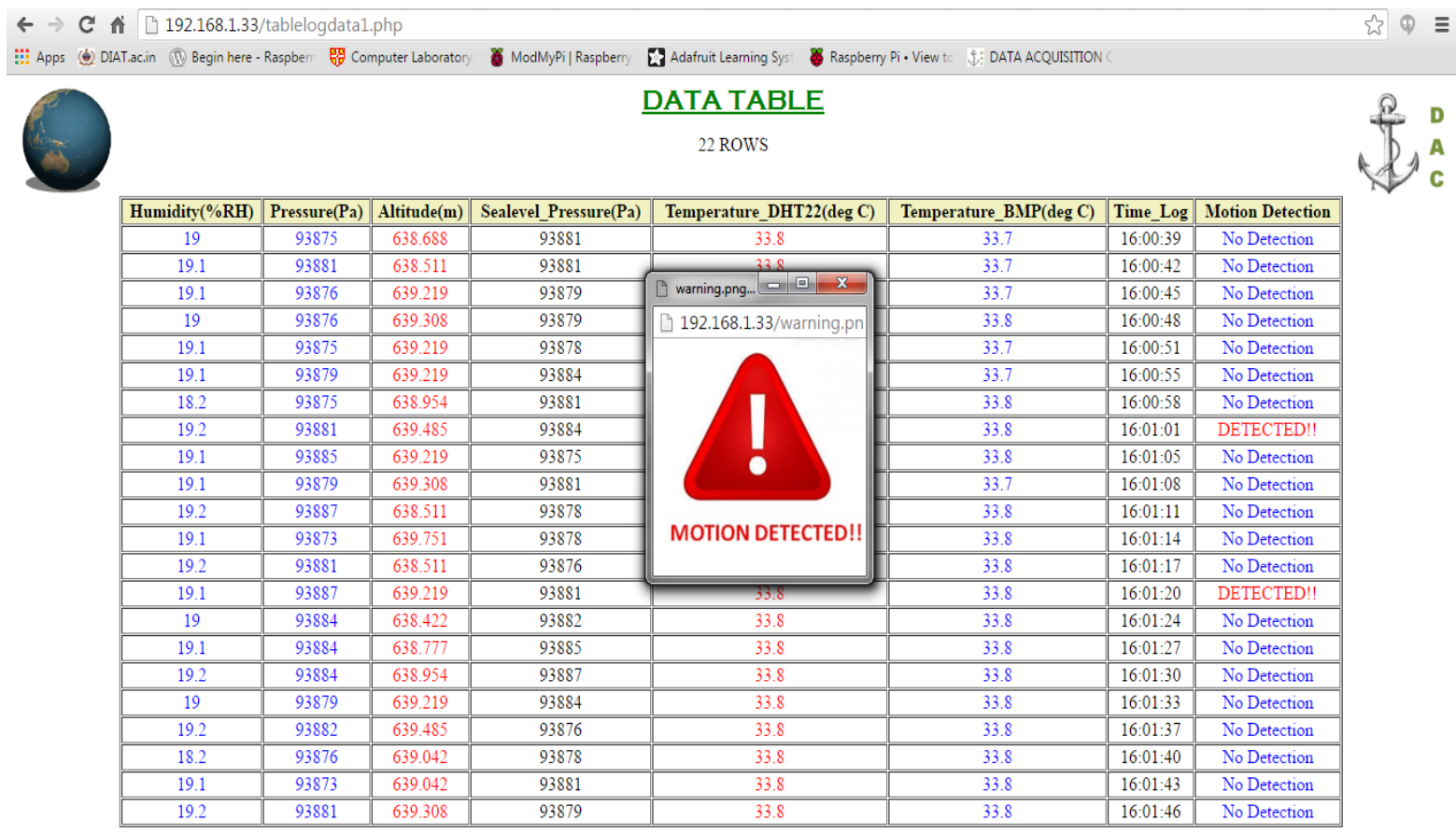

Fig.10. DAC Dashboard - Tabular view

All the graphs are generated using Libchart which is a free chart creation PHP library that is easy to use [17].

\section{4. $G U I$}

Data is displayed in an easily comprehendible manner in the form of a web based dashboard GUI. Readings within the limit are colour coded blue while the readings above the limit are highlighted in red.GUI allows the START/STOP of the data measurement program and also caters for total SHUT DOWN of the system. Provision for 
visual alarms, whenever a particular sensor reading crosses a pre-designated safe limit

\section{DAC PERFORMANCE}

The DAC was placed in a room to simulate a compartment onboard a marine vessel. Connectivity of the DAC to the existing WLAN setup was seamlessly achieved, also the Ethernet connectivity was checked via the RJ 45 Ethernet cable and no issues were observed in the setup. . The sensor reading program was initiated via the GUI and the measurements by the DAC were then compared with the measurements taken by independent sensors/ sources for validation. The independent measurement for temperature was taken using a traditional mercury-in-glass thermometer for measurement of room/compartment temperature. The altitude data was sourced from online websites [18] [19] which provided accurate altitude information for the geographical location. The humidity measurement was sourced via online sites [20] [21]. Although, the reference readings taken for altitude and humidity are from online sources and may not by $100 \%$ accurate, they do provide a yardstick for testing the performance of the DAC. A table depicting performance of the DAC for temperature, humidity and altitude is shown in table 2 below:-

Table 2. Sensor Performance

\begin{tabular}{|c|c|c|c|}
\hline Parameter & $\begin{array}{c}\text { Temperature } \\
\text { ( deg C) }\end{array}$ & Altitude (m) & Humidity \\
\hline $\begin{array}{c}\text { Independent } \\
\text { Measurement }\end{array}$ & 36.0 & 607 & $33 \%$ \\
\hline DAC Measurement & 35.7 & 604.8 & $32.5 \%$ \\
\hline$\%$ Error & $0.8 \%$ & $0.3 \%$ & $1.5 \%$ \\
\hline
\end{tabular}

For the PIR motion detection performance validation, various trials were conducted for motion detection at various ranges starting from within $1 \mathrm{~m}$ to up to $7 \mathrm{~m}$ range. Iterative process was used during the trial in which 10 instances of movement was undertaken each for $<1 \mathrm{~m}, 3 \mathrm{~m}$, $5 \mathrm{~m}$ and $7 \mathrm{~m}$ ranges respectively and the probability of detection $\left(\mathrm{P}_{\mathrm{d}}\right)$ based on these 10 iterations was calculated for each range. In all the trials, the sensitivity potentiometer of the PIR sensor was set for maximum i.e. $7 \mathrm{~m}$ range. It was observed that up to $3 \mathrm{~m}$ range, the $\mathrm{Pd}$ is around 0.9 and reduces to 0.6 for $6 \mathrm{~m}$ range. The probability of detection further reduces to 0.4 when an object is at the detection range limit of the sensor i.e. at $7 \mathrm{~m}$. The results are shown in Fig 11:-

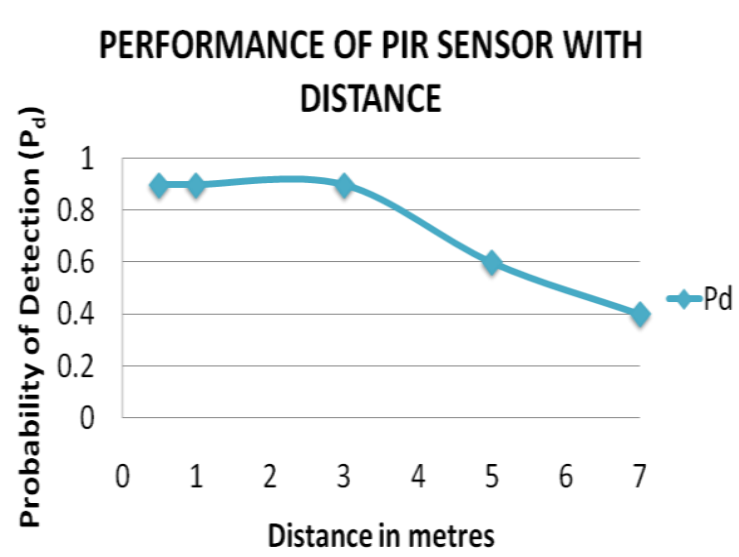

Fig.11. PIR Sensor Performance

On comparison of the measured readings, it has been found that the performance of the prototype DAC system is satisfactory with the measurement deviations observed being within the permissible accuracy limits of the individual sensors.

\section{CONCLUSION AND FUTURE WORK}

The genesis for design and development of the DAC for marine vessels both civil and military arose from the need to have further aid/means to enhance safety onboard ships. The performance of the DAC was successfully validated with favorable results by comparing the DAC measurements with the measurements taken from separate individual sensors. It was found that the error percentage was within $\pm 1.5 \%$ which is well within the permissible accuracy limit of the individual sensors. Validation for motion detection entailed an iterative process with repeated motion gestures at various distances from the sensor. Various readings were taken at different ranges in order to ascertain the probability of detection $\left(\mathrm{P}_{\mathrm{d}}\right)$ at various ranges. The sensor performance was satisfactory with a $\mathrm{P}_{\mathrm{d}}$ of 0.9 up to $3 \mathrm{~m}$ and 0.6 at a range of $5 \mathrm{~m}$. For compartments with dimensions larger than $5 \mathrm{~m}$, an additional DAC would need to be provisioned to cover the entire compartment and enhance the probability of detection.

The DAC offers flexibility, customization and can be easily deployed in compartments with LAN availability and also in areas without LAN by placing it to the nearest AP in order to transmit sensor readings over $\mathrm{Wi}-\mathrm{Fi}$ network. These systems are easily setup and advantageous as it utilizes the already existing LAN onboard ships which allows for savings in cost, space, weight of cabling and also does not involve any major piercings of bulkheads which may affect the gastight and watertight integrity of the ship. Further, it can be subjected to additions and alterations of sensors easily.

As the data is readily accessible by concerned personnel at any node on the LAN, it also helps in more efficient manpower management as it mitigates the requirement of taking physical rounds of restricted compartments. The successful testing and validation of the prototype DAC 
entails that the system may be readily deployed on civilian vessels however, for military platforms the system may need to be further ruggedized in order to conform to military standards such as EMI/EMC tests, vibration tests, environment testing to name a few

At present, the DAC can only detect motion and is able to give a visual indication with the aid of the red LED. Future scope can include integration of an audio alarm/buzzer in addition to a Pi camera which can be activated to click a snapshot the moment motion is detected. The clicked snapshot of the intruder/ trespasser can then be auto emailed to a fixed email address for instant notification/alert. The DAC can also be interfaced with an ultra sonic ranging module namely the HC SR04 sensor to detect distances and can thus be used as a collision avoidance system as well. A network of DACs each located in different compartments can be formed to cover all the relevant compartments onboard ship and the data can be displayed in a collated form on the web based dashboard which would go a long way to further enhance ship safety.

\section{ACKNOWLEDGMENT}

I am grateful to the faculty for their support, encouragement and timely course corrections and the facilities available which enabled us to see the fruit of our efforts in a time bound manner.

\section{REFERENCES}

[1] Raspberry $\mathrm{Pi}$ Official website [Online], http://www.raspberrypi.org/ Accessed on 28 Feb 16

[2] Hussein Kdouh, Gheorghe Zaharia, Christian Brousseau, Hanna Farhat, Guy Grunfelder and Ghaïs El Zein "Performance Analysis of a Hierarchical Shipboard Wireless Sensor Network", IEEE 23rd International Symposium on Personal, Indoor and Mobile Radio Communications - (PIMRC), 2012.

[3] Comdt (JG) Gaurav Sinha, M Tech Thesis "Modeling and analysis of ship based wireless sensor network", 2014. Department of Electronics Engineering, Defence Institute of Advanced Technology(DU) Pune.

[4] H.Farhat et al, "Double Directional Characterisation of Radio Wave Propagation through Metallic Watertight Doors on Board Ships", Vol. 48, Electronics Letters, 2012

[5] Vladimir Vujović, Mirjana Maksimović, Raspberry Pi as a Wireless Sensor Node: Performances and Constraints, 37th International Convention on Information and Communication Technology, Electronics and Microelectronics (MIPRO), May 2014,Croatia

[6] Harshada Chaudhari, Raspberry Pi Technology: A Review, International Journal of Innovative and Emerging Research in Engineering Volume 2, Issue 3, 2015 [7] $\begin{array}{lr}\text { DHT } & 22 \\ \text { https://www.sparkfun.com/datasheets/Sensors/Temperatur }\end{array}$ e/DHT22.pdf Accessed on 30 Oct 16

[8] HC-SR501 PIR MOTION DETECTOR datasheet. https://www.mpja.com/download/31227sc.pdf. Accessed on 02 Nov 16

[9] BMP180 Digital pressure sensor datasheet https://www.vssec.vic.edu.au/media/41229/BMP180datasheet.pdf. Accessed on 07 Mar 16

[10] BMP $\quad 180 \quad$ Sensor http://www.boschpresse.de/presseforum/pressdownload/text/PI7416.pdf?id
$=5192,2$ Accessed on 17 Apr 16.

[11] RTC Board. https://learn.adafruit.com/adding-a-real-timeclock-to-raspberry-pi/overview . Accessed on 07 May 16

[12] $\mathrm{SSH}$

Windows, https://www.raspberrypi.org/documentation/ remoteaccess/ssh/windows.md. Accessed on 19 Oct 16.

[13] Static IP address http://www.modmypi.com/blog/tutorialhow-to-give-your-raspberry-pi-a-static-ip-address Accessed on 19 Oct 16.

[14] Setting up PHPmyAdmin http://pimylifeup.com/raspberry-pi-mysql-phpmyadmin/ Accessed on 5 Feb 16.

[15] Elizabeth Naramore, Jason Gerner, Yann Le Scrournec, Jeremy Stolz, Michael k. Glass, "Beginning PHP5, Apache and MySQL Web Develpoment" 2005

[16] Janet Valade, "PHP and MySQL for Dummies - Second Edition" - Mar-2004

[17] Libchart, https://naku.dohcrew.com/libchart/pages /introduction/, Accessed on 15 Jan 16.

[18] http://www.mapcoordinates.net/en Accessed on 05 May 16

[19] https://www.daftlogic.com/sandbox-google-maps-findaltitude.htm Accessed on 05 May 16

[20] http://www.worldweatheronline.com/v2/weather.aspx?q= Khadakwasla,\%20India Accessed on 05 May 16

[21] http://www.weatheravenue.com/en/asia/in/maharashtra/kh adakwasla-weather-15-days.html Accessed on 05 May 16

\section{Authors' Profiles}

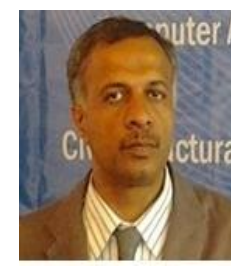

Dr. CRS Kumar, M.E., Ph.D, is Icurrently the Head of Department of Compuster Science in DIAT, Girinagar, Pune. He has a work experience of over 8 years. He is member of the Fellow of Institution of Electronics and Telecommunication Engineers, senior member of the IEEE and computer socitety of India. His areas of interest include Wireless Communication, Network Secuirty, Game Theory and Cognitive Radios Networks.

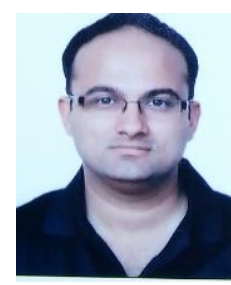

Aditya Thapliyal, received his B. Tech degree in Electrical and Electronics Engineering from JNUTU in 2007.He has worked in the field of IT and electronics systems. Currently he is undertgoing Masters in Technology programme in Defence Electronic Systems at DIAT, Girinagar Pune. His areas of interest include wirless communcations, computer networks and wireless sensors networks.

How to cite this paper: Aditya Thapliyal, CRS Kumar,"Development of Data Acquisition Console and Web Server Using Raspberry Pi for Marine Platforms", International Journal of Information Technology and Computer Science(IJITCS), Vol.8, No.11, pp.46-53, 2016. DOI: 10.5815/ijitcs.2016.11.06 